\title{
Coupled optical excitable cells
}

\author{
Alejandro M. Yacomotti* and Gabriel B. Mindlin \\ Departamento de Física, Facultad de Ciencias Exactas y Naturales, UBA, Ciudad Universitaria, \\ Pabellón I (1428) Buenos Aires, Argentina \\ Massimo Giudici and Salvador Balle \\ Departamento de Física Interdisciplinar, Instituto Mediterráneo de Estudios Avanzados, Univesidad de las Islas Baleares, E-07071 \\ Palma de Mallorca, Spain \\ Stephane Barland and Jorge Tredicce \\ Institut Non Linéaire de Nice, Centre National de la Recherche Scientifique, Unité Mixte de la Recherche 6618, 006560 Valbonne, France
}

(Received 4 April 2002; published 27 September 2002)

\begin{abstract}
In this work we investigate experimentally the dynamics of two coupled optical excitable cells, namely, two semiconductor lasers with optical feedback. We analyze the dynamics observed in terms of the statistical properties of the time series and in terms of the phase space reconstruction from the data. We build a model based on a simple set of deterministic equations (on a two torus) plus noise in order to capture the essential features of the dynamics observed. We discuss the validity of our theoretical results in terms of families of excitable systems and coupling terms.
\end{abstract}

DOI: 10.1103/PhysRevE.66.036227

PACS number(s): 05.45.-a, 42.65.Sf

\section{INTRODUCTION}

Many systems in nature present a behavior known as excitability. A system is said to be excitable whenever it has a stable stationary state and reacts, after a perturbation, in two qualitatively different ways: if the perturbation is smaller than a threshold, it evolves towards the initial state through a short excursion of its physical variables, while if the perturbation exceeds the threshold, the system returns to the stable state performing a large excursion of the variables [1].

Among the systems presenting this behavior in nature, one of the paradigmatical examples is the neuron. A large amount of research has focused on understanding which are the advantages of excitability in terms of computation ability, as well as to understand the dynamics of collective sets of excitable units $[2,3]$.

In recent years, a simple optical device was identified as excitable: the semiconductor laser with optical feedback [4]. Moreover, the onset of a characteristic dynamical state of this system (known as low frequency fluctuations) was discussed in terms of an excitable dynamical skeleton plus noise [5]. According to this paradigm, the erratic dropouts present at the onset of the low frequency fluctuations (LFF) are the consequences of the stochastic kicks that the system experiences, which can drive it beyond the threshold. Supporting this scenario, the statistical properties of the interspike time distributions (some of them nontrivial) can be fitted with a noise-driven excitable dynamical system [6]. Other elements of confidence in this scenario come from the recent observation of coherence resonance [7], and from the experimental analysis of the response to external modulation of a parameter [8-10]. Recently, other laser systems have been identified as excitable: broad-area semiconductor laser with optical

\footnotetext{
*Email address: yaco@df.uba.ar
}

injection leading to thermo-optical pulsation [11] and solidstate laser with saturable absorber [12].

The coupling of excitable systems has been addressed theoretically in the past, in an effort to understand the different kinds of synchronization regimes in arrays of noisedriven excitable units subjected to noise $[13,14]$. In particular, noise-induced synchronization regimes have been studied in two coupled excitable systems close to a codimension-two bifurcation (Andronov-homoclinic bifurcation). This leads to complex scenarios as a consequence of the dynamical evolution of the system in a four-dimensional phase space [15]. In such a case, the two-dimensional character of each cell is critical due to the proximity to the homoclinic bifurcation, as it occurs in the finite-dissipation excitable regime of the pendulumlike system discussed in Ref. [16]. As the dissipation of the cells is increased, a limit is reached for which the dynamics of each excitable unit is reduced to a onedimensional manifold (which can be mapped to a circle), and then the phase space of the coupled set becomes a two torus. In this strong dissipation limit, the dynamics of two coupled noise-driven excitable system can be fully understood in terms of a deterministic skeleton plus noise scenario, as it will be shown here.

In this work, we take the first steps towards the construction of arrays of optical excitable cells by coupling two semiconductor lasers with optical feedback. The coupling is realized optically, injecting a fraction of the intensity emitted by one excitable system into the other. The coupling strength is controlled independently from the amount of feedback for each laser. In this way, the excitable properties of each unit can be set independently from the degree of coupling between the two units.

In such conditions, we are able to address the following issues: How do two coupled semiconductor lasers with optical feedback behave? Can we explain this optical device in terms of simple models of coupled excitable cells? We orga- 


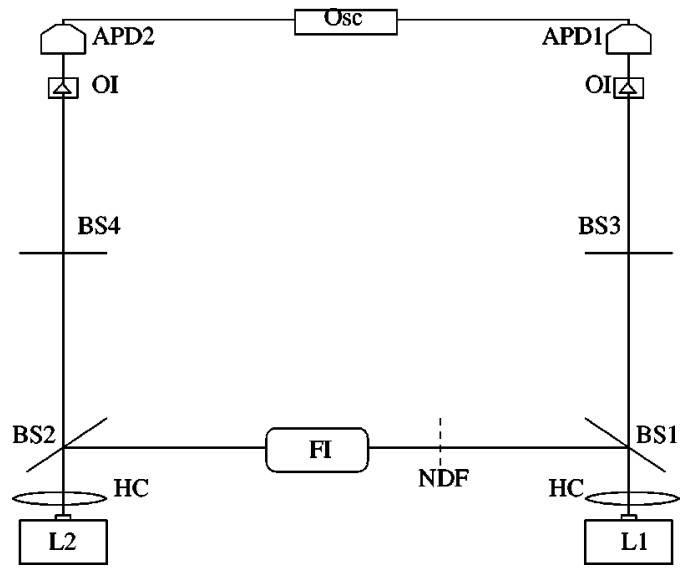

FIG. 1. Experimental setup for coupled excitable lasers. $L_{1,2}$, semiconductor lasers; HC, high numerical aperture collimators; BS's, beam splitters; OI's, optical isolators; APD's, avalanche photodiodes; NDF, neutral density filter; Osc, oscilloscope; FI, feedback isolator.

nize the work as follows. In Sec. II we describe the experiment. Section III contains the analysis of the experimental results. Section IV is devoted to the discussion of a simple model that captures the essential dynamical features of the experimental results. In Sec. V we interpret the experimental results in terms of the solutions of the model. In Sec. VI we present our conclusions.

\section{THE EXPERIMENT}

The experimental setup is shown in Fig. 1. It consists of two quantum-well edge emitters SDL $5400\left(L_{1}\right.$ and $\left.L_{2}\right)$ chosen from the same wafer in order to work with two mostly similar elements. The light at the output is collimated using two high numerical aperture collimators. The beams are divided by means of two beam splitters $\mathrm{BS}_{1}$ and $\mathrm{BS}_{2}$ of $50 \%$ of reflectivity. The reflected light on $\mathrm{BS}_{1}$ is directed towards $L_{2}$ and vice versa, which constitutes the coupling arm (distance between the lasers is $30 \mathrm{~cm}$, time delay, $1 \mathrm{~ns}$ ). The transmitted beams are focused onto two beam splitters $\left(\mathrm{BS}_{3}\right.$ and $\mathrm{BS}_{4}, R=25 \%$ ) in order to provide external feedback (external cavity length is $40 \mathrm{~cm}$ ). The transmitted beams of $\mathrm{BS}_{3}$ and $\mathrm{BS}_{4}$ are used for detection after passing through optical isolators, in order to prevent feedback from the measurement set. Two fast (bandwidth up to $2 \mathrm{GHz}$ ) photodiodes $\mathrm{APD}_{1}$ and $\mathrm{APD}_{2}$ are used together with a $500-\mathrm{MHz}$ digital scope (Lecroy 7200A) in order to monitor the signal output.

A neutral density filter (NDF) is used to set the coupling strength. Since we want to avoid spurious back reflection from one laser facet to the other one, we put in the coupling arm an optical device assuring unidirectional propagation of the injected beams. This device consists in two $\lambda / 4$-wave plates at $45^{\circ}$ with respect to the polarization direction of the lasers $\left(0^{\circ}\right)$, and a linear polarizer in between whose transmission axis is set at $0^{\circ}$. This device allows for injection from one laser into the other while it cuts down any back reflection of the injected beam.

The solitary laser thresholds were measured to be $J_{t h, 1}$
$=15.35 \mathrm{~mA}$ and $J_{t h .2}=15.23 \mathrm{~mA}$. The excitable regime is obtained, properly setting the pumping current and the feedback level of each laser $[4,6]$. We prepared our lasers in this regime fixing the feedback to a level yielding to threshold reduction of $\approx 15 \%$, while pumping currents were varied in the range $\pm 0.05 J_{t h}$ around the threshold value. In this range, as a general characteristic, the lasers exhibit almost constant intensity traces with sporadic (less that 10 in $100 \mu$ s for the highest current), randomly distributed excitable pulses and the pulsation rate tends to increase as the pumping current is increased.

\section{RESULTS}

In this section we present the experimental results for the excitable lasers with bidirectional coupling.

We choose as observables the intensity output of the lasers, $I_{1}$ and $I_{2}$. The control parameters are the pumping parameters $\left(p_{i} \equiv J_{i} / J_{t h, i}\right)$ and the coupling intensity normalized to the intensity emitted by the lasers $(\beta)$. In this work, $\beta$ will be kept smaller than 0.05 .

In order to analyze the effects of the coupling in our system, for $\beta=0$ we set $J_{1}$ and $J_{2}$ in such a way that the rate of excitable pulses obtained in each laser is the same. The homogeneous situation cannot be obtained simply by setting $J_{1}=J_{2}$ due to unavoidable small construction differences in the lasers or to differences in the feedback levels.

When the coupling is enabled, and $\beta$ is sufficiently large (typically larger than $10^{-3}$ ), the drop frequency dramatically increases up to $1-5 \mathrm{drops} / \mu \mathrm{s}$, depending on the pumping current and the coupling strength.

In Fig. 2(a) we present the time series for medium coupling, $\beta=0.007$, and pumping parameters $p_{1}=1.04$ and $p_{2}$ $=1$. We observe large segments of constant intensity, randomly distributed in time. In addition, the pulse amplitude does not present important fluctuations. Notice that there are some pulses in $I_{1}$ that are synchronized with pulses in $I_{2}$ within a time window of the order of the pulse width. In addition, there are some pulses in $I_{1}\left(I_{2}\right)$ that have no counterpart in $I_{2}\left(I_{1}\right)$. It is important to remark that, when a pulse in one laser is accompanied by the occurrence of a pulse in the other, the time lag between pulses can be much larger than $1 \mathrm{~ns}$, which is the trip time between the lasers [see Fig. 2(b)].

In order to describe the time distributions of pulses, we perform interpulse time histograms. We define $\mathbf{t}_{\mathbf{1}}$ $=\left\{t_{1}(1), \ldots, t_{1}(N)\right\}$ as the vector of the time values at which the pulses $(1, \ldots, N)$ in $I_{1}$ occur, and $\mathbf{t}_{2}$ $=\left\{t_{2}(1), \ldots, t_{2}(M)\right\}$ as the corresponding vector of $I_{2}$. The interpulse time distributions for each subsystem is calculated as the histograms of $t_{1}(i)-t_{1}(i-1)$ (with $1<i \leqslant N$ ) and $t_{2}(j)-t_{2}(j-1)$ (with $1<j \leqslant M$ ), respectively. The results are shown in Fig. 3(a) in continuous and dashed lines for $I_{1}$ and $I_{2}$. We first note that the distributions of the individual signals show the characteristics of a distribution of randomly distributed events; we remark that the exponential tail for long time intervals and the cutoff for short-time intervals resemble statistical distributions present in noise-driven excitable systems, where the cutoff is related to the so-called 

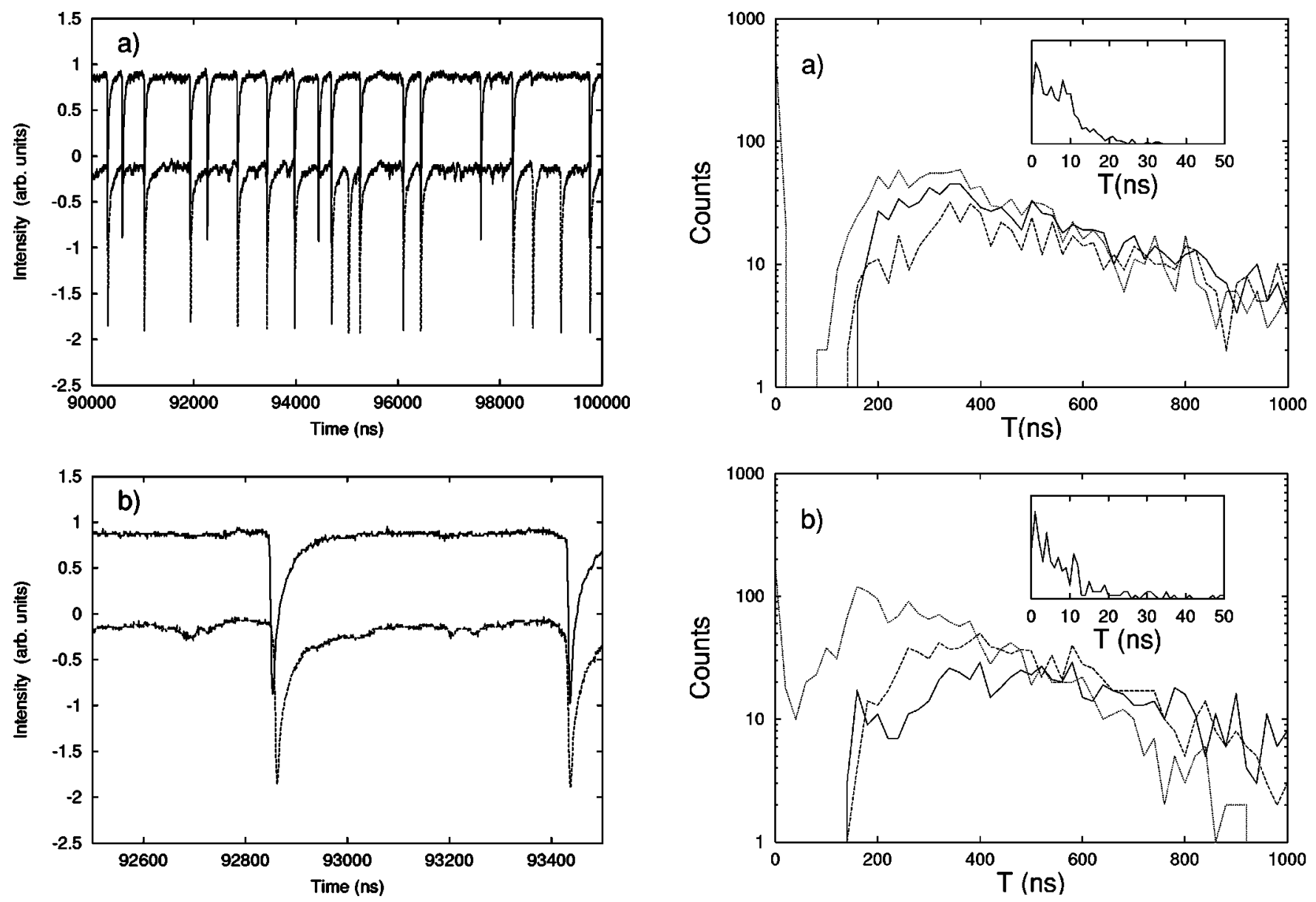

FIG. 2. A segment of typical experimental time traces for coupled excitable lasers (medium coupling). The signals are vertically shifted for clarity. Up (continuous line), $I_{1}$; down (dashed line), $I_{2}$. (b) Zoom of (a). The control parameters are $p_{1}=1.04$, $p_{2}=1$, and $\beta=0.007$ (see definitions of $p$ and $\beta$ in the text).

refractory time [16]. We also notice that interpulse time distributions of both subsystems are quite similar in shape.

In order to address the degree of correlation between pulses in $I_{1}$ and those in $I_{2}$ we build a new vector $\mathbf{t}$ $=\{t(1), \ldots, t(N+M)\}$ with the elements of $\mathbf{t}_{\mathbf{1}}$ and $\mathbf{t}_{\mathbf{2}}$, in increasing order. In other words, $\mathbf{t}$ contains the successive times where a pulse occurs, regardless of whether it comes from $L_{1}$ or $L_{2}$. The histogram of $t(k)-t(k-1$ ) (with $1<k$ $\leqslant N+M$ ) is shown in Fig. 3(a) by the dotted line. From now on we will refer to this curve as "interpulse time histogram of the joint series." We find that there is one wide peak with almost the same exponential decay for long times as in the distributions of the individual series. At short-time intervals, we observe the existence of a peak $\approx 10 \mathrm{~ns}$ wide [see inset of Fig. 3(a)]. It is worth noting the absence of events between 30 and $70 \mathrm{~ns}$, which determines an "empty region" between the two peaks.

In Fig. 3(b) we show the time interval distributions for the weak coupling regime, $\beta=0.005$. It is important to mention that the pulsation frequency decreases as $\beta$ is decreased. Thus, in order to compare these distributions with the me-

FIG. 3. Experimental time interpulse $(T)$ histograms of the intensity time series of $L_{1}$ (continuous line) and $L_{2}$ (dashed line); dotted line, the joint series distribution (see definition in the text). (a) Medium coupling $(\beta=0.007)$; (b) low coupling $(\beta=0.005)$. Inset: zoom for short-time intervals (the vertical scale is linear). The total number of pulses in each laser is $\approx 950$.

dium coupling regime we increase the pumping currents $\left(p_{1}=1.06\right.$ and $\left.p_{2}=1.03\right)$ so that the pulse rate is kept constant while coupling is decreased. As a result, each signal in Fig. 3(b) has approximately the same number of total events $(\approx 950)$ as in Fig. 3(a).

We can see two main differences between the joint series distributions of Fig. 3(b) (weak coupling) and Fig. 3(a) (medium coupling): as the coupling is decreased, the empty region between the two peaks in the distribution vanishes with a slight broadening of the short-time peak, and the slope of the long-time tail is increased (in absolute value) with respect to the individual distributions.

A useful tool for getting information on the system's dynamics is the reconstruction of the phase space through timedelay embeddings of the time series. In Fig. 4 we show timedelay embeddings of the time series of both lasers. The fact that the trajectories almost collapse to a one-dimensional manifold allows us to infer that a good choice for the variables describing the trajectories in the plane could be the phase variables defined as the angles $\left(\theta_{1}, \theta_{2}\right)$ of the vectors pointing from an adequate center to any point on the trajec- 


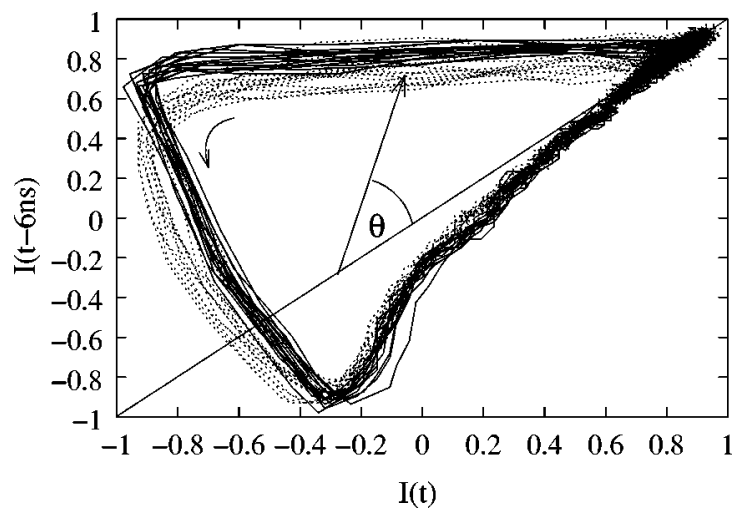

FIG. 4. Time-delay embedding of the time series. Continuous line: $I_{1}$; dashed line: $I_{2}$. The phase variable $\theta$ is constructed as the angle pointing from an adequate center to the trajectories.

tories in the plane. By this choice of the variables we obtain an embedding on a two torus.

We then build the phase space $\left(\theta_{1}, \theta_{2}\right)$ as follows.

(1) For each peak in $I_{1}$ at time $t_{1}(i)$, the occurrence of a peak in $I_{2}$ is checked within a time window, i.e., we look for $t_{2}(j)$ such that

$$
t_{1}(i)-t_{r} \leqslant t_{2}(j) \leqslant t_{1}(i)+t_{r},
$$

for some $j, t_{r}$ being the refractory time that is the minimum interpulse interval found in the individual series (which coincides with the time spent by $\theta_{1,2}$ to perform one complete excursion).

(2) If (1) is verified for some $j$, the event is then called "synchronization event" of type $S_{1}, S_{2}$, or $S_{p}$ depending on whether $t_{2}(j)-t_{1}(i)>1 \mathrm{~ns}, t_{1}(i)-t_{2}(j)>1 \mathrm{~ns}$, or $\mid t_{2}(j)$ $-t_{1}(i) \mid \leqslant 1$ ns (we recall that $1 \mathrm{~ns}$ is the coupling time, i.e., the trip time between lasers). The latter is called "in-phase synchronization event." In other words, a synchronization event is of type $S_{1}\left(S_{2}\right)$ when a pulse in $I_{1}\left(I_{2}\right)$ anticipates its counterpart in $I_{2}\left(I_{1}\right)$ within the refractory time, with a time lag longer than the coupling time. Moreover, an $S_{p}$ event is defined as the synchronization pair having a time lag equal to or smaller than the coupling delay. Each event is displayed in the $\left(\theta_{1}, \theta_{2}\right)$ phase space between $\max \left[t_{1}(i), t_{2}(j)\right]-t_{r}$ and $\max \left[t_{1}(i), t_{2}(j)\right]+t_{r}$.

(3) If condition (1) is not verified for any $j$, then the event is called "localization event" since for this $i$ th pulse there is no counterpart in the other subsystem. The phase space representation of this type of event is trivial since it corresponds to one complete phase excursion in $\theta_{1}$ with $\theta_{2}$ remaining in its stationary value.

The phase portraits corresponding to the synchronization events are displayed in Figs. 5 and 6. From Fig. 5(a) to Fig. 5(c) we have decreased the coupling strength, while the pumping currents have been increased in order to maintain the same number of total events $(\approx 950)$. Figures $5(\mathrm{~b})$ and 5(c) correspond to the parameter values of Fig. 3(a) and 3(b), respectively. In Figs. 6(a)-6(c) we explore lower coupling levels, while the pumping current values follow the same sequence as in Fig. 5; as a consequence, in Figs. 6(a)-6(c) the number of events is $\approx 450$.
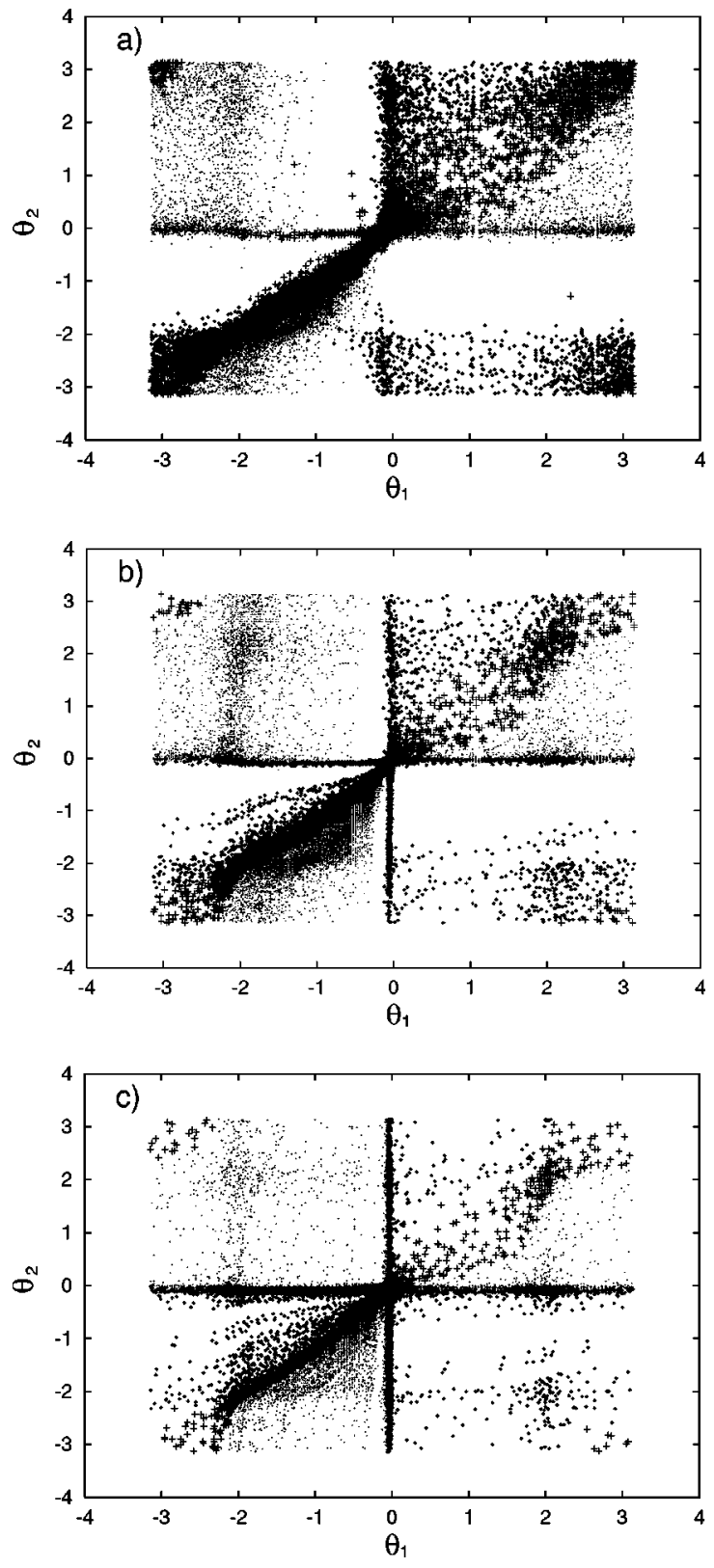

FIG. 5. Experimental phase embeddings for high pulsation rate $(\approx 2$ pulses $/ \mu \mathrm{sec})$ and decreasing coupling strength. $\theta_{1,2}$ come from Fig. 4. Only synchronization events are displayed. The trajectories on the phase space are grouped in families of different synchronization types: crosses, "in-phase synchronization" (type $S$ ); thick dots, $I_{1}$ anticipating $I_{2}$ (type $S_{1}$ ); dots, $I_{2}$ anticipating $I_{1}$ (type $S_{2}$ ). (a) $\beta=0.02$, (b) $\beta=0.007$, and (c) $\beta=0.005$.

We now concern ourselves with a qualitative description of the reconstructed phase portraits. We first define the fraction of localized events (in $L_{1}$ ) as $\gamma \equiv$ (localization no.)/(total no.).

In Fig. 5(a) a nonvisited region in the phase space can be identified between the regions filled with $S_{1}$ and $S_{2}$ events. This nonvisited region shrinks in Fig. 5(b) and almost disappears in Fig. 5(c). In addition, the $\gamma$ fraction increases from Fig. 5(a) to 5(c) we calculated $\gamma=0.28$ in Fig. 5(a), 0.41 in 5 (b), and 0.58 in 5(c). Similar tendencies in the evolution of the phase space and localization fractions are observed from 

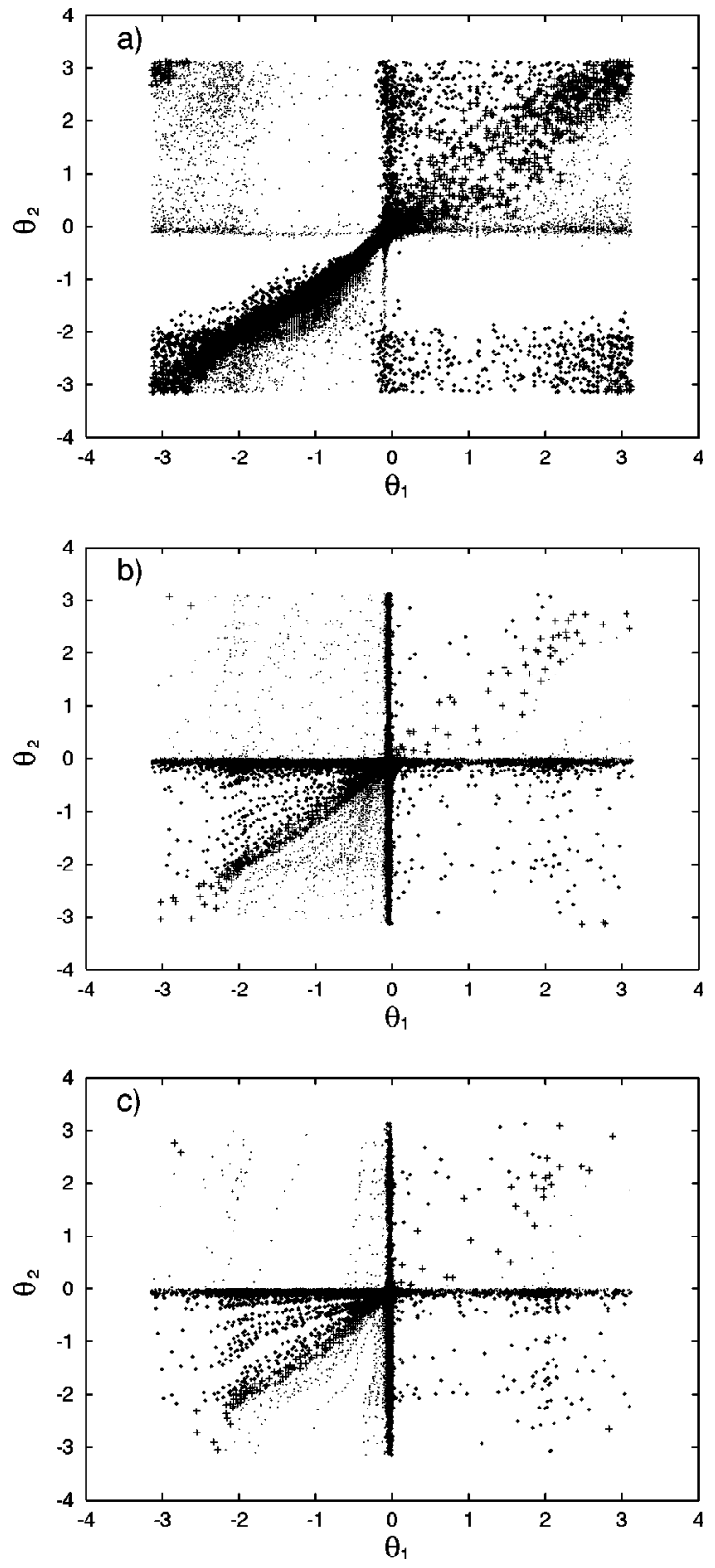

FIG. 6. Experimental phase embeddings for low pulsation rate $(\approx 1$ pulse $/ \mu \mathrm{sec})$ and decreasing coupling strength. (a) $\beta=0.01$, (b) $\beta=0.003$, and (c) $\beta=0.001$. Definitions of the symbols are in Fig. 5 .

Figs. 6(a) to 6(c), which correspond to a different number of total events. In Fig. 6(b), however, the unvisited region almost disappears. In this case the $\gamma$ values were calculated to be $\gamma=0.17$ in Fig. 6(a), 0.54 in 6(b), and 0.74 in 6(c).

It is important to note that there are no systematic crossings between branches of trajectories corresponding to different types of events, which allows us to build confidence in our low-dimensional embedding of the signals.

It is also worth mentioning that the quantity of $S_{p}$ events decreases as the coupling is reduced. As a general conclusion we state that, for a given pulse rate, the nonvisited region in the phase space between the regions filled with $S_{1,2}$ events contracts as the coupling strength is decreased, together with an increase in localized events. The statistical correspondence in this mechanism relies on the distributions of the joint series shown in Fig. 3. The presence of nonvisited regions in the phase portraits is reflected by empty time regions between the two peaks in the histograms of the joint series [see Figs. 3(a-b)].

\section{THE MODEL}

We are now concerned with the description of the experimental results by means of a simple dynamical model. Our strategy is to establish the essential model features needed to duplicate the main experimental observations. The requirements are the following.

(1) The dynamical model has to be representative within a family of excitable systems. In the case of systems close in parameter space to a linear singularity (i.e., close to a local bifurcation), the procedure to find the simplest equation representative of the phenomenon is algorithmic. This simple equation is known as normal form. In order to describe excitability, this procedure cannot be applied since it involves a global property of the flow (a reinjection of the dynamics into the neighborhood of the stationary state after a large excursion within the available region of the variables of the problem).

(2) The model has to be as simple as possible in terms of phase space dimensionality. We recall that the experimental data seem to be well described by two variables (the phase variables $\theta_{1}$ and $\theta_{2}$ ). Therefore, we choose 2 as the dimension of the deterministic skeleton of our model.

(3) The coupling terms to be proposed have to be representative, within a family of coupling terms, of the observed dynamical evolution. This means that, given two excitable cells, different coupling terms lead to different phase space structure, and therefore to distinct qualitative changes as the parameters are changed (i.e., bifurcations). Thus, the coupling terms have to be chosen in such a way that they are able to fit the experimental phase space structure, together with the statistical properties of the time series.

(4) Since the synchronization is a consequence of interaction between the subsystems via the pulses, it is desirable to fit the observed pulse shape with the model in order to capture the dynamics of the mutual excitation. In our case, we deal with asymmetrical pulses, i.e., pulses that have an ignition slope faster than the recuperation tail, as can be observed from the experimental data [see Fig. 2(b)].

Points (1)-(3) are linked to the topological properties of the model solutions, while point (4) attempts to fit the metric properties of the experimental phase portraits that are related to some statistical observables.

Our approach to fulfilling the requirements is based on Adler's equation. This equation plays well the role of a dynamical system that is simple and displays excitability. This system describes the dynamics of an angular variable $\theta$ according to 


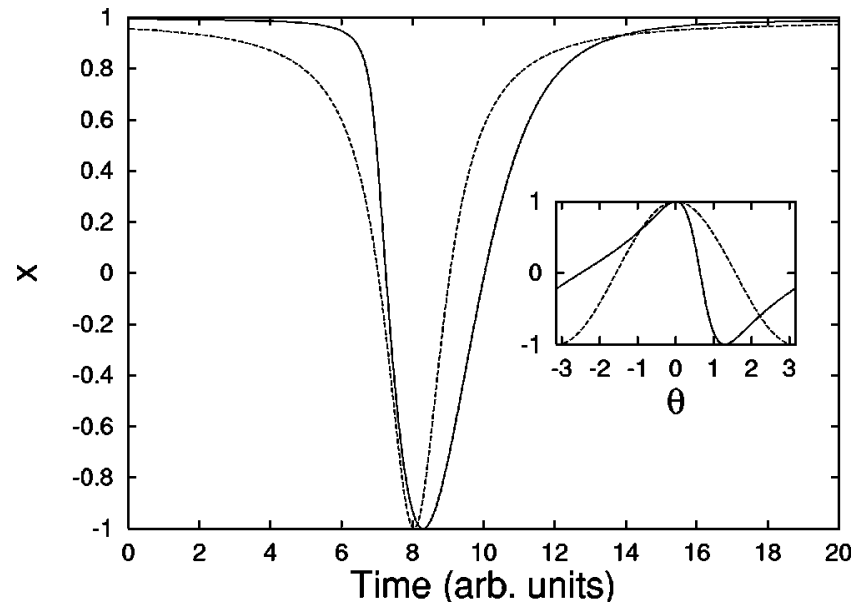

FIG. 7. Solutions of model (3). Continuous line, $a=0.8$; dashed line, $a=0$ (Adler's equation). Inset: vertical axis, $f(\theta)$. $x \equiv \cos (\theta)$. Notice the asymmetry of the pulses when $a>0$, relative to the different rates in the ignition and recuperation slopes of the vector field, contrasting to the Adler's case.

with $\mu \in \mathcal{R}$. For $\mu<1$, the system presents two fixed points, one unstable and another one stable $\left[\right.$ at $\left.\theta_{u, s}= \pm \arccos (\mu)\right]$. For $\mu>1$, the system displays no fixed points, and the dynamics consists in self-sustained oscillations. The transition between these two regimes is known as Andronov bifurcation. Recent studies showed that the statistical properties of the interspike time distribution of the dropouts in a semiconductor laser with optical feedback at the onset of LFF can be explained in terms of the system being close to an Andronov bifurcation in the presence of noise. For this reason, we use this system as the inspiration for a simple caricature to model the behavior of each laser.

The excitable pulses emerging from (2) are symmetric in shape, as shown in Fig. 7. In order to account for requirement (4), we introduce a slight modification of this equation such that we can achieve asymmetric pulses. The new model reads

$$
\dot{\theta}=\mu-f(\theta),
$$

with

$$
f(\theta)=\frac{1}{f_{0}} \frac{\sin \left(\theta+\theta_{0}\right)}{1+a \cos \left(\theta+\theta_{0}\right)}
$$

and

$$
\begin{gathered}
f_{0}=\frac{1}{\sqrt{1-a^{2}}}, \\
\theta_{0}=\arccos (-a) .
\end{gathered}
$$

The constants $f_{0}$ and $\theta_{0}$ are such that $f(0)=1$ is a maximum. Figure 7 shows the function $f(\theta)$ for two parameters $a$, together with the dynamical evolution of $x \equiv \cos (\theta)$, which we suggest to be related to the measured variable in the experiment (the intensity). In the case $a=0$, Adler's equation is duplicated and the pulse shape is symmetric. As $a$ is in- creased, the function $f(\theta)$ becomes nonsymmetric with respect to the $\theta=0$ axis. For instance, $a=0.8$ corresponds to two different rates for the dynamical evolution of the pulse: the negative slope of $f(\theta)$ governs the ignition of the pulse, while the recuperation is ruled by the positive slope before returning to the stable state (for $\mu<1$ ), which is smoother than the escape. These two distinct slopes for the ignition and the recuperation lead to the asymmetry of the pulse, as can be clearly seen in Fig. 7 .

Model (3) (for $a>0$ ) is topologically equivalent to Adler's equation $(a=0)$. In particular, there also exist two fixed points for $\mu<1$, which now read

$$
\begin{aligned}
\theta_{s, u}= & \arccos \left\{\frac{a \mu^{2}}{f_{0}^{2}+\mu^{2} a^{2}}[-1\right. \\
& \left.\left. \pm \sqrt{1+\left(f_{0}^{2}-\mu^{2}\right)\left(f_{0}^{2}+\mu^{2} a^{2}\right) /\left(\mu^{4} a^{2}\right)}\right]\right\}-\theta_{0},
\end{aligned}
$$

and for $\mu>1$ the system develops self-oscillations after the Andronov bifurcation that takes place at $\mu=1$.

To describe the dynamics of two optical excitable cells, we begin by coupling Eq. (3) for the two cells in the manner discussed in Ref. [17],

$$
\begin{aligned}
& \dot{\theta}_{1}=\tilde{\mu}-f\left(\theta_{1}\right)+\epsilon \sin \left(\theta_{2}-\theta_{1}\right), \\
& \dot{\theta}_{2}=\tilde{\mu}-f\left(\theta_{2}\right)+\epsilon \sin \left(\theta_{1}-\theta_{2}\right),
\end{aligned}
$$

where the functional form of the coupling corresponds to the translation into angular variables of a simple restitutive coupling in the Euclidean variables $(\epsilon>0)$. The coupling terms are representative, within a family, of a phase space structure consistent with what is observed in the experiment. We also modified the parameter $\mu$ introducing a new parameter $\tilde{\mu}$ $\equiv \mu+c \quad \epsilon$, with $c$ a constant to be determined. This means that the effect of the coupling is reflected not only in the restitutive coupling terms, but there is also a constant term proportional to the coupling which is added.

Before describing the solutions of model (8) for $a>0$, it is of fundamental importance to remark that the resulting phase space structure is topologically equivalent to that obtained from Adler's equation $(a=0)$. In other words, we find the same organization of the invariant manifolds, and the same bifurcations as the coupling parameter is changed. Working with $a>0$ matches some metric features present in the experimental results, with respect to the curvature of the invariant manifolds on the two tori, and also matches more closely the statistical properties that arise as noise is added to the system. Besides that, the bifurcation points take different values with respect to the case $a=0$. Despite these differences, all the results we will show here concerning the model (8) for $a>0$ are equivalent to those with $a=0$.

We begin the description of the dynamical responses of the system (8) by noticing that $\theta_{1}=\theta_{2}$ is an invariant set. Within it, both cells behave as an excitable system (3). Hence, two fixed points exist, namely, $\theta_{\mathbf{u}, \mathbf{s}}=\left(\theta_{u, s}, \theta_{u, s}\right)$, re- 

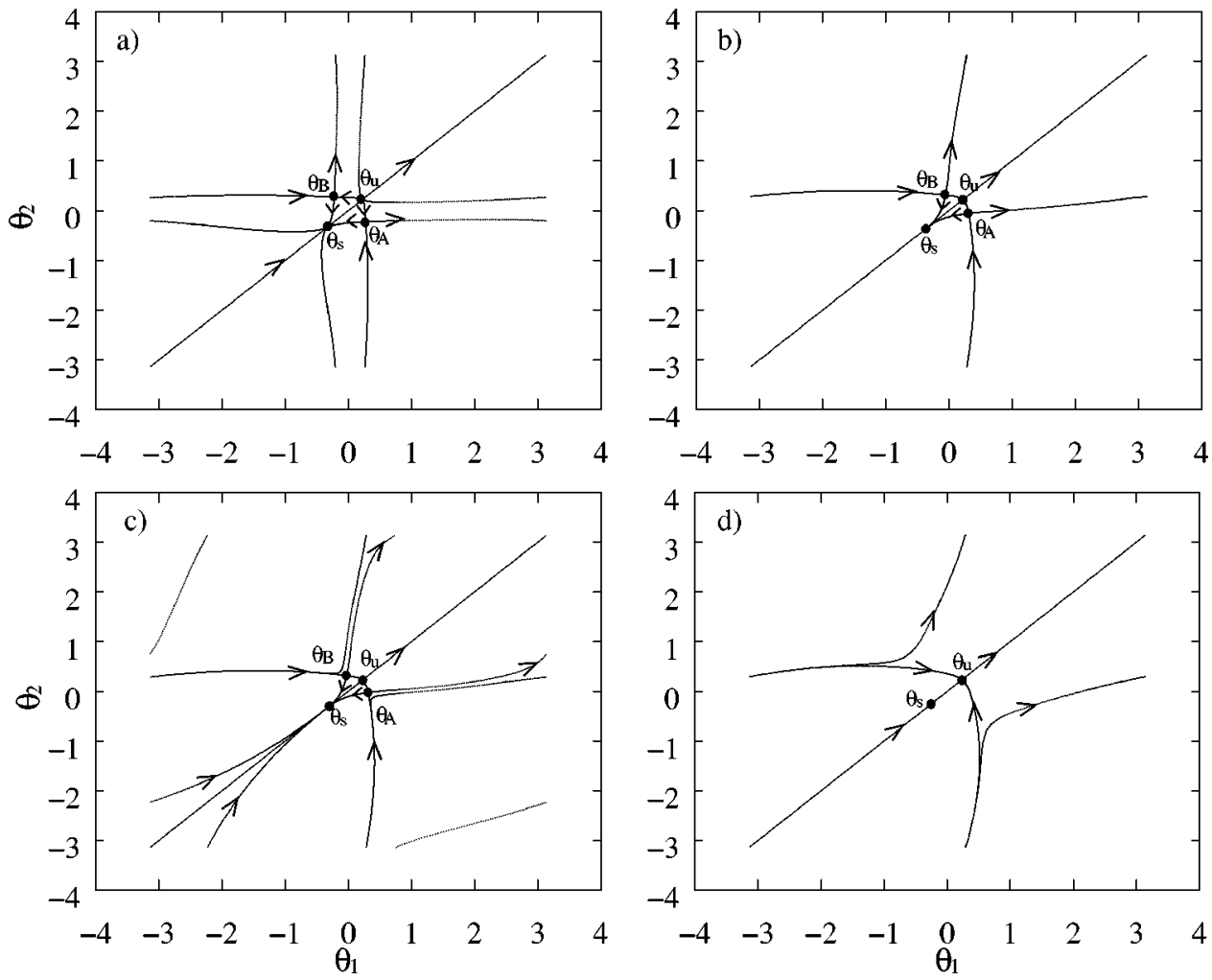

FIG. 8. Invariant manifolds of the fixed points in the model of coupled excitable cells [Eq. (8)]. The parameter $\tilde{\mu}$ is kept fixed at 0.9 , and $a=0.8$. (a) $\epsilon=0.1$; (b) $\epsilon$ $\approx 0.27=\epsilon_{h}$ (at the heteroclinic bifurcation); (c) $\epsilon=0.3$, where the saddles $\theta_{A, B}$ coexist with an unstable periodic orbit; (d) $\epsilon=0.55$, beyond the pitchfork bifurcation that takes place at $\epsilon_{c} \approx 0.49$. placing $\mu \rightarrow \tilde{\mu}$ in the Eq. (7). The other eigenvectors of these fixed points will be perpendicular to the invariant direction (by symmetry). The fixed point at $\theta_{1}=\theta_{2}=\theta_{s}$ is always a stable node, while the fixed point at $\theta_{1}=\theta_{2}=\theta_{u}$ will be unstable in the transverse direction as long as $\epsilon<\epsilon_{c} \equiv-[a$ $\left.+\cos \left(\theta_{u}+\theta_{0}\right)\right] /\left[2 f_{0}\left[1+a \cos \left(\theta_{u}+\theta_{0}\right)\right]^{2}\right.$, becoming a repulsor. In this regime, two saddle fixed points exist outside the invariant subspace, namely, $\theta_{\mathbf{A}, \mathbf{B}}[$ see Fig. 8(a)]. The stable manifolds of these fixed points are part of the unstable manifold of $\theta_{\mathbf{u}}$. The fixed points $\theta_{\mathbf{A}, \mathbf{B}}$, calculated numerically, exist for $\epsilon<\epsilon_{c}$, and they are born in the direction perpendicular to $\theta_{1}=\theta_{2}$.

Let us discuss the different dynamical scenarios that can be found, as $\epsilon$ is increased, for $\tilde{\mu}$ fixed. For $\epsilon<\epsilon_{c}$, the four fixed points described above coexist in the phase space. At $\epsilon=\epsilon_{h}<\epsilon_{c}$, a heteroclinic bifurcation takes place, in which a branch of the unstable manifold of the fixed point $\theta_{\mathbf{A}}$ becomes one of the branches of the stable manifold of $\theta_{\mathbf{B}}$, and a branch of the unstable manifold of $\theta_{\mathbf{B}}$ becomes one of the branches of the stable manifold of $\theta_{\mathbf{A}}$ [see Fig. 8(b)]. At this value of the coupling parameter, an unstable periodic orbit is born (with infinite period).

Notice that before the heteroclinic bifurcation, no trajectory along the unstable manifold of $\theta_{\mathbf{A}}\left(\theta_{\mathbf{B}}\right)$ performs an excursion in $\theta_{2}\left(\theta_{1}\right)$. After the heteroclinic bifurcation, in turn, a branch of the unstable manifold of $\theta_{A}$ feeds the stable manifold of the stable fixed point after a complete excursion along both $\theta_{1,2}$ [see Fig. 8(c)]. The same occurs for the fixed point $\theta_{\mathbf{B}}$.

At $\epsilon=\epsilon_{c}$ the two fixed points outside the invariant axis collide with the fixed point at $\theta_{\mathbf{u}}$ in a pitchfork bifurcation. Further increasing the coupling, the only fixed points are located along the invariant axis, and coexist with a periodic orbit that winds around the phase space [Fig. 8(d)].

In previous works $[4,6]$, the dynamical evolution of the intensity of semiconductor lasers with optical feedback, in the LFF regime, was explained in terms of a deterministic skeleton and noise. Even the detailed structure of the interspike time distribution and its evolution as the parameters were changed could be explained in this way. In the same spirit, we interpret the experimental results described in the preceding section in terms of the deterministic structure of the flow of two coupled excitable cells plus noise.

Let us study then the solutions of

$$
\begin{aligned}
& \dot{\theta}_{1}=\tilde{\mu}-f\left(\theta_{1}\right)+\epsilon \sin \left(\theta_{2}-\theta_{1}\right)+\sqrt{2 D} \xi_{1}(t), \\
& \dot{\theta}_{2}=\tilde{\mu}-f\left(\theta_{2}\right)+\epsilon \sin \left(\theta_{1}-\theta_{2}\right)+\sqrt{2 D} \xi_{2}(t),
\end{aligned}
$$

where $\xi_{1}(t)$ and $\xi_{2}(t)$ are independent white noise terms with zero mean and variance one, and $D$ accounts for the amplitude of the stochastic terms.

We performed numerical simulations for parameter values of the deterministic part of the equations above corresponding to the situations displayed in Figs. 8(a) and 8(c). In Fig. 9 we show the time series obtained with parameters of the deterministic part of the equations as in Fig. 8(c), with parameter $\tilde{\mu}$ taken to be 0.98 . Notice that even if the deterministic parts of the equations have a unique fixed point attractor, the evolution of the stochastically driven system is quite complex.

Following the same data analysis protocol as in Sec. III, we build an interpulse time histogram for the independent series as well as for the mixed series for two coupling pa- 


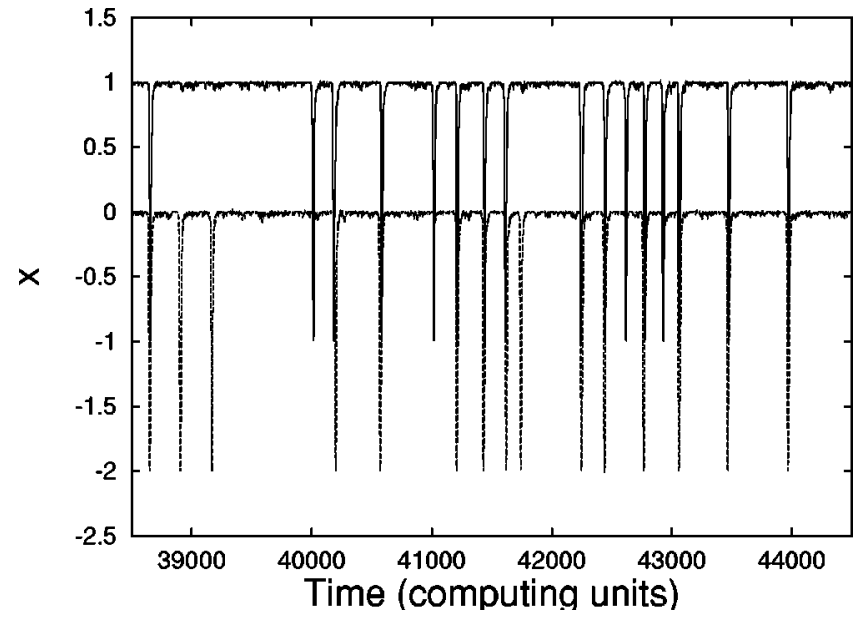

FIG. 9. Time series of the model of coupled excitable cells with noise [Eq. (10)]. $x \equiv \cos (\theta)$. The traces are vertically shifted for clarity. Up (continuous line), $x_{1}$; down (dashed line), $x_{2}$. Parameters are $\tilde{\mu}=0.98, D=3 \times 10^{-3}$, and $\epsilon=0.16\left(\epsilon>\epsilon_{h} \approx 0.13\right)$, corresponding to the deterministic regime of Fig. 8(c). Notice the complexity of the solution, even when the deterministic part of the equation has a unique fixed point attractor.

rameters: high coupling $\left[\epsilon_{c}>0.16>\epsilon_{h}\right.$, corresponding to the case of Fig. 8(c)], shown in Fig. 10(a), and low coupling $\left[0.05<\epsilon_{h}\right.$, corresponding to the deterministic skeleton of Fig. 8(a)], shown in Fig. 10(b). For these two regimes we keep $\tilde{\mu}=0.98$ fixed, which is achieved choosing $c=0.182$, and $\mu=0.951$ in the high coupling regime, and $\mu=0.971$ in the low coupling regime.

The distributions have the same features as in the experiment: (i) the long-time decay slope in the mixed series distribution is larger than those of the individual series for low coupling, while for large coupling they remain close; (ii) there is an empty time interval between the two peaks of the joint series distribution, which vanishes as the coupling is decreased; (iii) the peak corresponding to short times in the joint series distribution broadens as the coupling is decreased.

In Fig. 11(a), we display the projection of the numerical integration for $\epsilon=0.16$ in the $\left(\theta_{1}, \theta_{2}\right)$ space, with the same method used in Sec. III, together with the deterministic invariant manifolds of the fixed points. We recall that the events displayed in this phase portrait are of type $S$ (synchronization). Besides, the fraction of localized events was calculated to be $\gamma=0.16$.

We begin the interpretation of the phase space by noting that the excitable threshold is the union of the stable manifolds of $\theta_{\mathbf{A}}$ and $\theta_{\mathbf{B}}$. Let us focus on an event that is triggered in the region $\theta_{2}<\theta_{1}$. The events triggered close to $\theta_{A}$ will evolve following closely the branch of the unstable manifold of $\theta_{A}$ corresponding to $\dot{\theta}_{1}>0$ (which is contractive in the transverse direction). This is evidenced by a high density of flux around this invariant manifold. This manifold departs from the unstable periodic orbit (it changes direction rapidly around $\left.\theta_{1} \approx \pi\right)$, giving rise to an unvisited region in the phase portrait. These events are of type $S_{1}$ ( $\theta_{1}$ anticipating $\theta_{2}$ ). The same occurs for the $S_{2}$ events. There are also events
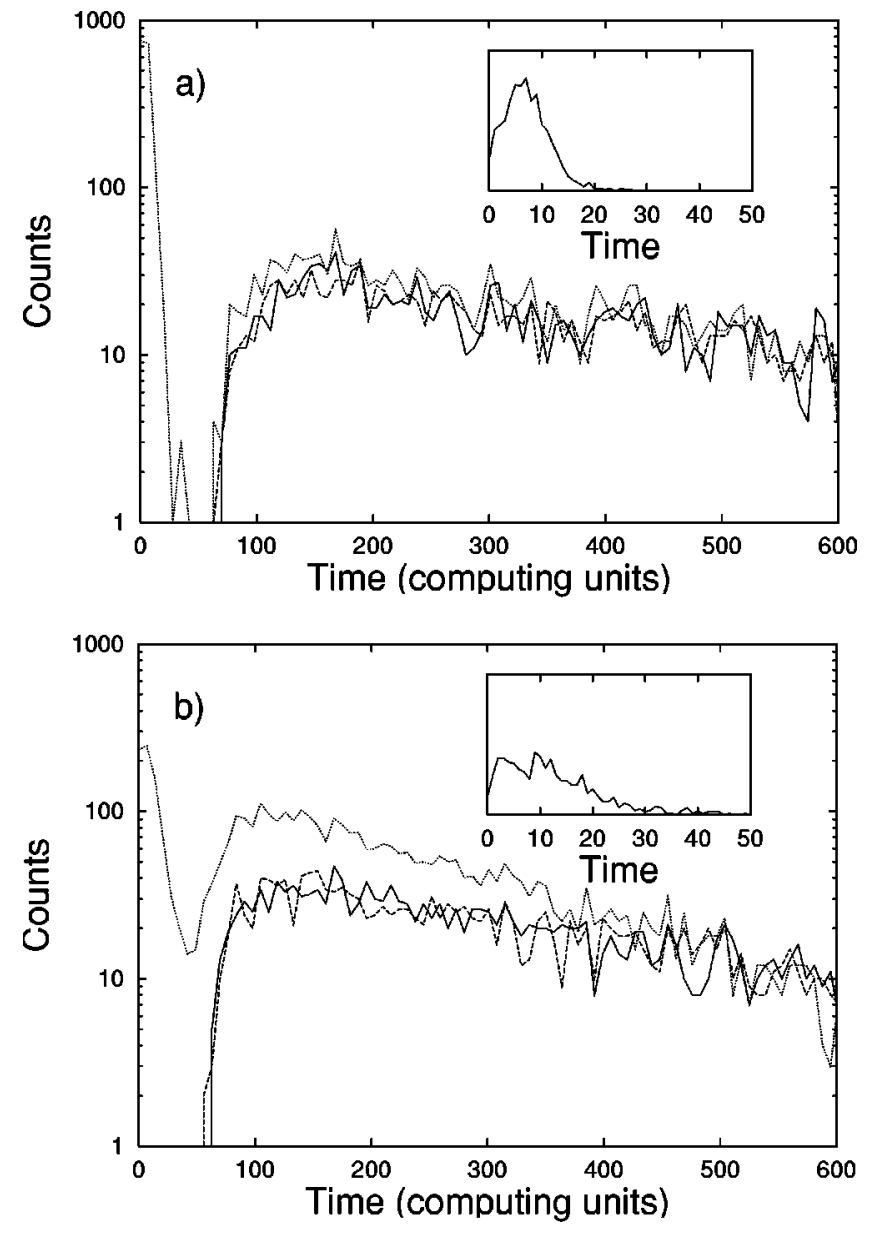

FIG. 10. Theoretical time interpulse $(T)$ histograms calculated from the model of coupled excitable cells plus noise. Continuous (dashed) line, interpulse distribution of $\cos \left(\theta_{1(2)}\right)$; dotted line, the joint series distribution. (a) high coupling $(\epsilon=0.16)$; (b) low coupling $(\beta=0.05)$. Inset: zoom for short-time intervals (the vertical scale is linear). The other parameters are $\tilde{\mu}=0.98$ and $D=3$ $\times 10^{-3}$. The total number of pulses in each subsystem is $\approx 2000$.

that are triggered close to $\theta_{\mathbf{u}}$, which evolve around the invariant axis in the form of $S_{p}$ synchronized pulses. In Fig. 11(b) we show the localization events. These events correspond to trajectories that, after crossing the excitable threshold, also cross the unstable periodic orbit before being attracted towards the unstable manifold of the saddle. These noise-induced crossings of an unstable invariant set occur rarely, and then the fraction of localized events is small. It is worth noting that the unstable periodic orbit sets a deterministic bound for the maximum time delay between $S$ pulses, i.e., the maximum time lag for $S$ events corresponds to a trajectory evolving along the unstable periodic orbit.

Let us now analyze the situation for low coupling, $\epsilon$ $=0.05$. The phase portrait of the synchronized events is shown in Fig. 11(c), together with the underlying deterministic skeleton. In this case, the $\gamma$ fraction was calculated to be 0.63 .

We can explain the large fraction of localized events from the deterministic skeleton. As in the preceding case, the system only develops complete excursions in $\theta_{1}$ or $\theta_{2}$ as long as 

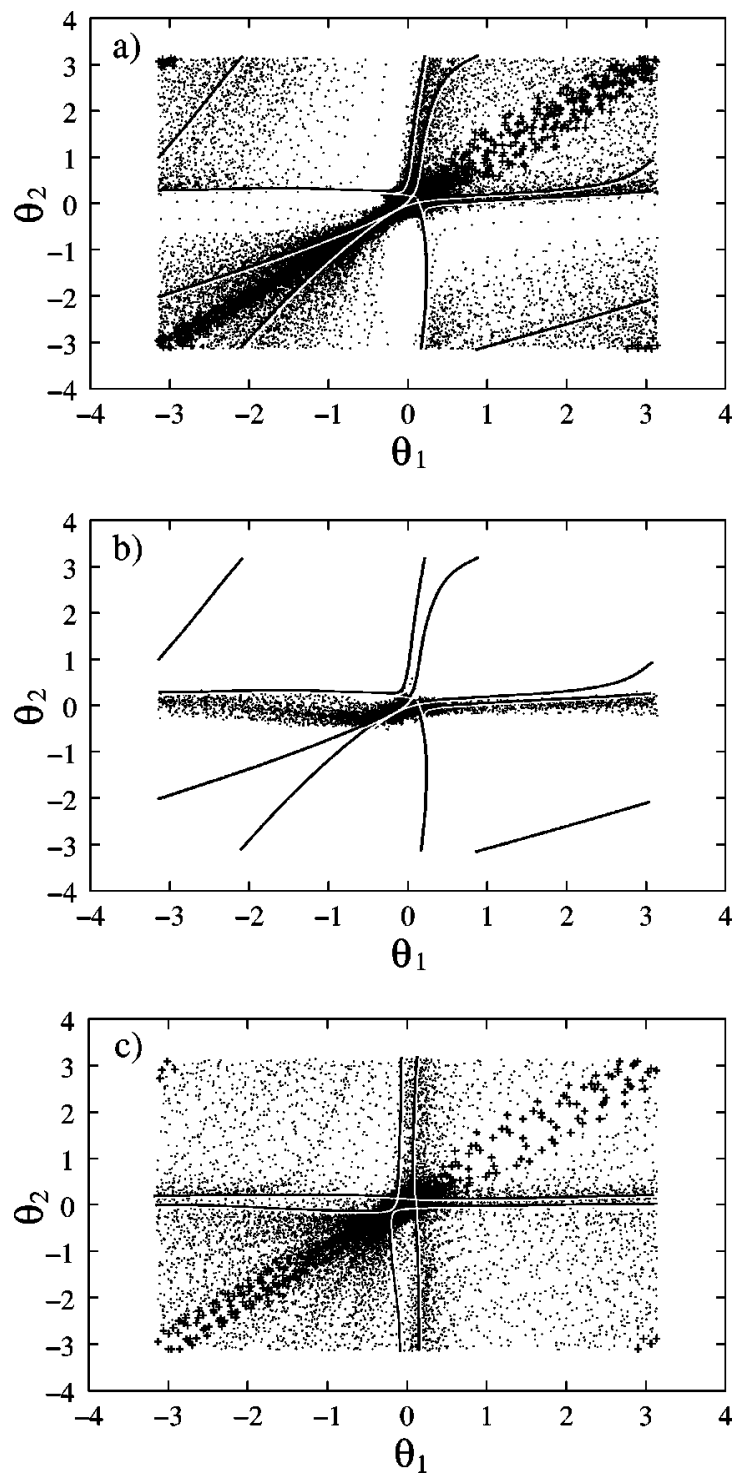

FIG. 11. Theoretical phase embeddings for $\tilde{\mu}=0.98$ and $D=3$ $\times 10^{-3}$. (a) Synchronization events (symbols) for $\epsilon=0.16$ (high coupling). Families for different synchronization-types are calculated with the same method as in Fig. 5. Crosses, "in-phase synchronization" (type $S$ ); dots, $S_{1,2}$ events. (b) Localization events (dots) for $\epsilon=0.16$. (c) Synchronization events (symbols) for $\epsilon$ $=0.05$ (low coupling). Lines: the invariant manifolds of the deterministic part of the equations.

it crosses the union of the stable manifolds of the saddles. Taking this into account, and since the unstable manifolds of $\theta_{\mathbf{A}, \mathbf{B}}$ feed the stable manifold of the stable fixed point after only one excursion in $\theta_{1}$ or $\theta_{2}$, the trajectories will mostly end at the node in an event of type $L$, yielding a large $\gamma$ fraction. Moreover, the $S$ events represented in Fig. 11(c) can also be interpreted in terms of the geometry of the phase space. In order to do this, let us focus on an event of type $S_{1}$. Due to the effect of noise, the system will eventually cross the threshold close to the fixed point $\theta_{\mathbf{A}}$. Now, if the noise causes the trajectory to cross the (larger) branch of the stable manifold of $\theta_{\mathbf{B}}$ before it reaches the node, then it will evolve up to it performing a subsequent complete excursion in $\theta_{2}$, which constitutes the type $S_{1}$ event. We can also explain the existence of events of type $S_{p}$, with trajectories crossing the threshold close to $\theta_{\mathbf{u}}$.

Let us study the features of the phase portrait. Notice that the trajectories of Fig. 11(c) are spread all over the phase space. In contrast to the case for $\epsilon>\epsilon_{h}$, we see that there are no unvisited regions, i.e., there are no empty regions between those filled with $S_{1}$ and those filled with $S_{2}$ events. This feature can be interpreted with the deterministic skeleton plus noise scheme. Specifically, it was shown that the existence of a nonvisited region is a consequence of the presence of an unstable periodic orbit that rapidly rejects the unstable branch of a saddle, giving rise to an $S$ event. Now, this periodic orbit no longer exists, and therefore there is no underlying mechanism capable of separating in phase space the regions filled with $S_{1}$ trajectories from those filled with $S_{2}$ trajectories.

\section{COMPARISON WITH EXPERIMENT}

In order to compare the experimental results with the dynamical model, we establish a relation between the control parameters $p$ and $\beta$ with the parameters of the model, $\mu$ and $\epsilon$. For a laser with feedback, it has already been shown that the excitable threshold is essentially controlled by the pumping parameter $(p)$, which is related to the parameter of the Andronov bifurcation $[4,6]$ given by $\mu$ in the case of model (3). Therefore, we relate $p \approx \mu$. Now, in the case of two coupled excitable lasers, we have found that the pulse frequency for the two cells depends on both the pumping parameter and the coupling parameter, namely, the frequency increases with both $p$ and $\beta$. In model (10), the excitable threshold is essentially governed by $\tilde{\mu}$ (at least in the range of parameters used in this work) and therefore the pulsation frequency depends on $\tilde{\mu}$. If we relate $\beta \approx \epsilon$, then the proposal $\tilde{\mu}=\mu+c \epsilon$ becomes justified if $c$ is properly chosen. In particular, we have already mentioned that our choice $c$ $=0.182$ yields $\mu=0.951$ in the case $\epsilon=0.16$, and $\mu$ $=0.971$ in the case $\epsilon=0.05$, in such a way that $\tilde{\mu}=0.98$. For the these values of $\mu$, the solitary excitable cells $(\epsilon=0)$ are almost stable, i.e., they show very few drops on a long time scale, exactly as in the experimental case.

Let us compare Figs. 11(a) and 11(c) with Figs. 5(b) and 5(c). In both cases, a decrease in the coupling strength has the effect of filling the unvisited regions. Furthermore, we suggest that the region of high density of $S_{1}$ flux in Fig. 5(b) (for $\theta_{1}<0$ and $\theta_{2}>0$ ) can be the fingerprint of the presence of the unstable manifold of the saddle, as can be seen in Fig. 11(a). In addition, the fraction of localized events increases as the coupling strength is decreased, and the order of magnitude of $\gamma$ is comparable between experiment and model. The same behavior can be observed in Figs. 6(a)-6(c).

In conclusion, the main effect of reducing the coupling strength of two coupled excitable cells in the presence of noise is to shrink the unvisited region in phase space, together with increasing the fraction of localized pulses. This can be completely understood in terms of a deterministic skeleton plus noise as a consequence of a global heteroclinic 
bifurcation that changes the topology of the (larger) branches of the unstable manifolds of the saddles. As a consequence of the bifurcation, these branches change from evolving on the torus performing an excursion in both variables, to performing an excursion in a single variable. The bifurcation annihilates the unstable periodic orbit responsible for the "repulsion" of trajectories in the phase space, and also for the low fraction of localized events that arise (for $\epsilon>\epsilon_{h}$ ) as rare noise-induced crossings of the periodic orbit.

Finally, it is important to say that the simple existence of an unstable periodic orbit is not a sufficient condition for the existence of nonvisited regions. It is also required that this unstable set should be sufficiently repulsive in the transverse direction. In our case, this is manifested by the rapid separation of the unstable manifold from the unstable periodic orbit, as seen for $\epsilon=0.16$ [Fig. 11(a)]. From the point of view of the pulse shape, this can be seen as a consequence of the two different rates in the ignition and in the recuperation of the excitable excursion: as two pulses become synchronized with a time lag, the larger starts to develop faster compared with the slow recuperation tail of the leader, which is manifested as the sudden inflection of the unstable manifold of the saddles. If we consider the coupled Adler equation [ $a$ $=0$ in (3)], the fact that the pulse shape is symmetric makes it necessary to increase the coupling parameter much further above the heteroclinic bifurcation point in order to separate sufficiently the unstable manifold from the unstable periodic orbit. As a consequence, both sets become far apart at any point, and then the localization fraction decreases dramatically. In fact, it turns out that in trying to fit the nonvisited regions by means of two coupled Adler equations, the localization fraction falls dramatically below 0.01 , which is not consistent with the experimental results, where we obtained approximately $20 \%$ of localization events together with empty regions in the phase space (for large coupling). By contrast, in the model we build to fit the experiment, we are able to find values of $\gamma$ close to the experimental one for large coupling, coexisting with empty regions in the phase portraits. Indeed, as shown in Fig. 11(a), focusing on $S_{1}$ events, the unstable manifold of the saddle stays close to the unstable periodic orbit up to $\theta_{1} \approx \pi$ when it is suddenly repelled upwards. This situation makes it possible to have an important $\gamma$ fraction as early crossings of the unstable periodic orbit, compatible with large nonvisited regions. According to these considerations, requirement (4) of Sec. IV to build a suitable model is justified.

\section{CONCLUSIONS}

In this work we have shown that two lasers with feedback in the excitable regime, coupled by mutual optical injection, can be interpreted in terms of two coupled excitable cells. This was achieved by studying phase variables of the intensity dropouts, together with the interpulse time distributions, in comparison with a model of two modified coupled Adler equations in the presence of noise. The agreement of the experimental results with the model was verified in two distinct ways: from the fingerprints of deterministic invariant sets in the phase portraits, and from the evolution of statistical features, such as the fraction of localized events (one pulse in one cell with no counterpart in the other) and histograms of time between pulses, as the coupling parameter was varied.

Even if our model was built to fit the features of the experiment, the geometry of the phase space is equivalent to two coupled Adler equations. Then we can claim that our results can be adapted to a large class of excitable systems with an Andronov bifurcation. Moreover, we are working on adapting the simple dynamical mechanisms described here to explain the case of other excitable paradigms such as the FitzHugh-Nagumo equations.

We verified that the underlying dynamical processes involved in our paradigm are valid for a large family of coupling terms. The angular coupling used in this work is derived from coupling terms in the Cartesian coordinates for the Euclidean representation of an Adler-like equation. Writing the system (8) in Cartesian coordinates (see Ref. [17] for the Adler case), it is easy to demonstrate that the coupling terms present in Eq. (8) are derived from restitutive coupling terms in $\dot{x}$ and $\dot{y}$. We would like to remark that the resulting angular coupling terms turn out to be the same for direct Euclidean coupling terms of the form $\left(\dot{x}_{1}, \dot{y}_{1}, \dot{x}_{2}, \dot{y}_{2}\right)=\mathbf{f}$ $+\boldsymbol{\epsilon}\left(x_{2}, y_{2}, x_{1}, y_{1}\right), \mathbf{f}$ being the vector field for the uncoupled system. Furthermore, a different angular coupling but with the same bifurcations as Eq. (8) arises from direct Euclidean coupling of only the excitatory variable $y$. In summary, we claim that the basic results presented here, in terms of underlying dynamical structure, belong to a large class of excitable systems and coupling terms, provided the dissipation of the two-dimensional excitable cell is large enough; the work by $\mathrm{Hu}$ and Zhou [15] points in the direction of coupled excitable cells in the presence of noise with finite dissipation.

\section{ACKNOWLEDGMENTS}

This work was partially funded by FOMEC and CONICET (Argentina). G.M. and A.Y. want to thank M. C. Eguia for enlightening comments. A.Y. thanks P. Colet for helpful discussions.
[1] J.D. Murray, Mathematical Biology (Springer, New York, 1990).

[2] J. Maddox, What Remains to be Discovered: Mapping the Secrets of the Universe, the Origin of Life, and the Future of the Human Race (Touchstone Books, 1999).
[3] W.S. McCulloch and W.H. Pitts, Bull. Math. Biophys. 5, 115 (1943).

[4] M. Giudici, C. Green, G. Giacomelli, U. Nespolo, and J.R. Tredicce, Phys. Rev. E 55, 6414 (1997).

[5] M.C. Eguia, G.B. Mindlin, and M. Giudici, Phys. Rev. E 58, 
2636 (1998).

[6] A.M. Yacomotti, M.C. Eguia, J. Aliaga, O.E. Martinez, G.B. Mindlin, and A. Lipsich, Phys. Rev. Lett. 83, 292 (1999).

[7] G. Giacomelli, M. Giudici, S. Balle, and J.R. Tredicce, Phys. Rev. Lett. 84, 3298 (2000).

[8] F. Marino, M. Giudici, S. Barland, and S. Balle, Phys. Rev. Lett. 88, 040601 (2002).

[9] J.M. Mendez, R. Laje, M. Giudici, J. Aliaga, and G.B. Mindlin, Phys. Rev. E 63, 066218 (2001).

[10] J.M. Mendez, J. Aliaga, and G.B. Mindlin (unpublished).
[11] S. Barland, Ph.D. thesis, Université de Nice Sophia Antipolis (2001).

[12] M.A. Larotonda, A. Hnilo, J.M. Mendez, and A.M. Yacomotti, Phys. Rev. A 65, 033812 (2002).

[13] C. Kurrer and K. Schulten, Phys. Rev. E 51, 6213 (1995).

[14] B. Hu and Ch. Zhou, Phys. Rev. E 61, R1001 (2000).

[15] B. Hu and Ch. Zhou, Phys. Rev. E 63, 026201 (2001).

[16] M.C. Eguia and G.B. Mindlin, Phys. Rev. E 61, 6490 (2000).

[17] M. Sigman and G.B. Mindlin, Int. J. Bifurcation Chaos Appl. Sci. Eng. 10, 1709 (2000). 